\title{
Next-generation sequencing approach to hyperCKemia
}

\author{
A 2-year cohort study
}

Anna Rubegni, MD, Alessandro Malandrini, MD, Claudia Dosi, MD, Guja Astrea, MD, Jacopo Baldacci, BS, Carla Battisti, MD, Giulia Bertocci, BSc, M. Alice Donati, MD, M. Teresa Dotti, MD, Antonio Federico, MD, Fabio Giannini, MD, Salvatore Grosso, MD, Renzo Guerrini, MD, Sara Lenzi, MD, Maria A. Maioli, MD, Federico Melani, MD, Eugenio Mercuri, MD, Michele Sacchini, MD, Simona Salvatore, MD, Gabriele Siciliano, MD, Deborah Tolomeo, MD, Paola Tonin, MD, Nila Volpi, MD, Filippo M. Santorelli, MD, and Denise Cassandrini, PhD

Neurol Genet 2019;5:e352. doi:10.1212/NXG.0000000000000352

\section{Abstract \\ Objective}

Next-generation sequencing (NGS) was applied in molecularly undiagnosed asymptomatic or paucisymptomatic hyperCKemia to investigate whether this technique might allow detection of the genetic basis of the condition.

\section{Methods}

Sixty-six patients with undiagnosed asymptomatic or paucisymptomatic hyperCKemia, referred to tertiary neuromuscular centers over an approximately 2-year period, were analyzed using a customized, targeted sequencing panel able to investigate the coding exons and flanking intronic regions of 78 genes associated with limb-girdle muscular dystrophies, rhabdomyolysis, and metabolic and distal myopathies.

\section{Results}

A molecular diagnosis was reached in 33 cases, corresponding to a positive diagnostic yield of $50 \%$. Variants of unknown significance were found in 17 patients $(26 \%)$, whereas 16 cases (24\%) remained molecularly undefined. The major features of the diagnosed cases were mild proximal muscle weakness (found in 27\%) and myalgia (in 24\%). Fourteen patients with a molecular diagnosis and mild myopathic features on muscle biopsy remained asymptomatic at a 24-month follow-up.

\section{Conclusions}

This study of patients with undiagnosed hyperCKemia, highlighting the advantages of NGS used as a first-tier diagnostic approach in genetically heterogeneous conditions, illustrates the ongoing evolution of molecular diagnosis in the field of clinical neurology. Isolated hyperCKemia can be the sole feature alerting to a progressive muscular disorder requiring careful surveillance.

\author{
Correspondence \\ Dr. Santorelli \\ filippo3364@gmail.co
}




\section{Glossary}

CK = creatine kinase EFNS = European Federation of Neurologic Societies; HPO = Human Phenotype Ontology; LGMD = limb-girdle muscular dystrophy; NGS = next-generation sequencing; ULN = upper limit of normal; VUS = variants of unknown significance.

Creatine kinase (CK) levels can be mildly and transiently increased as a result of muscle injury or exercise, even in healthy individuals. Persistent elevation of serum CK (termed hyperCKemia) is defined, according to European Federation of Neurological Societies (EFNS) guidelines (ean.org/ Guideline-Reference-Center.2699.0.html), as the presence of serum CK values beyond 1.5 times the upper limit of normal (ULN) in least 2 measurements, ${ }^{1}$ and it is a common reason for referrals to specialized neuromuscular centers.

Increased serum CK can be present in the absence of obvious clinical signs. ${ }^{2}$ In individuals with a normal neurologic examination, the condition is termed asymptomatic or isolated hyperCKemia, and it can signal the presence of several unsuspected metabolic, cardiac, rheumatic, or endocrine conditions. When no underlying cause is found, it is referred to as idiopathic hyperCKemia. The clinical management of idiopathic hyperCKemia is unclear; although clinically asymptomatic, affected patients are potentially susceptible to malignant hyperthermia. ${ }^{3}$

In asymptomatic hyperCKemia, definition of the correct diagnosis may be time consuming, and success is not guaranteed. Next-generation sequencing (NGS) has recently been proposed as a cost-effective strategy for the molecular diagnosis of inherited neuromuscular disorders. ${ }^{4}$ The efforts to define the molecular etiology in hyperCKemia come from the need to refine follow-up avoiding unnecessary examinations and to improve counseling in the family. We set out to explore whether NGS might allow detection of the molecular basis of hyperCKemia, addressing this question in a consecutive series of adults and children recruited at 7 Italian tertiary neuromuscular centers over an approximately 2 -year period.

\section{Methods}

\section{Standard protocol approvals, registrations, and patient consents}

This study was approved by the Tuscany Regional Pediatric Ethics committee. All the procedures complied with the Helsinki Declaration of 1975. Genetic studies and muscle biopsies were performed with written informed consent. All participants (including parents or legal guardians in case of minor patients) were provided pre- and post-test genetic counseling as routine in our neurogenetic clinics.

\section{Patients and study design}

Over an approximately 2-year period (May 2016-August 2018), 66 patients presenting with hyperCKemia (meeting the EFNS criteria) were consecutively referred to the neurology, pediatric, or neuropediatric units of 7 Italian tertiary neuromuscular centers for clinical and diagnostic purposes. All 66 met the inclusion criteria for our study: (1) persistent serum CK elevation at rest and (2) values higher than twice the ULN on at least 2 occasions after refraining from muscular exercise for at least 72 hours before CK measurement. For each patient, we collected clinical and laboratory data and the results of familial segregation analyses and previous genetic tests. MRI scans of thigh and calf muscles were performed in 26 patients, EMG in 26, and muscle biopsy in 57, in all cases using routine clinical methods. ${ }^{5}$ Before this study, we had performed multiplex ligation-dependent probe amplification analysis in all the patients to exclude multiexon rearrangements in the DMD gene and tested the levels of acid alpha-glucosidase from dried blood spots ${ }^{6}$ to detect possible undiagnosed late-onset Pompe disease. Patients with borderline enzyme values or biallelic mutations in the GAA gene were not included in this study. DNA samples were analyzed at a single center.

None of the 66 individuals had a medical history of anesthesiarelated complications or a family history of cardiovascular events. Any family history of neuromuscular disorders or mild muscle complaints was carefully recorded. Occasional causes of hyperCKemia, such as malignancies, drug and alcohol abuse, rheumatic, thyroid, and parathyroid disorders, infections, and hematologic diseases, were all excluded. All patients underwent routine serum chemistry, including serum myoglobin measurement. No participant was under statin treatment or taking other drugs potentially capable of inducing hyperCKemia.

\section{NGS workflow and sequencing analyses}

We used the SureSelect technology (Agilent, Santa Clara, CA) and SureDesign software (earray.chem.agilent.com/ suredesign/) to design a multiexon amplicon panel containing a total of 78 genes known to be associated with limb-girdle muscular dystrophies (LGMDs), rhabdomyolysis, and metabolic and distal myopathies ${ }^{7}$; the panel spanned more than $259 \mathrm{Mbp}$, with gene coverage $>99 \%$. To analyze the data obtained from our study, we used a routine bioinformatic pipeline ${ }^{8}$ that adopts the Ingenuity Variant analysis suite (Qiagen, apps.ingenuity.com). To assign pathogenicity, we set up a precise Alissa (Agilent) pipeline using the following criteria: a sequence quality score greater than 30 , a read depth greater than 30 , and rare occurrence in publicly available polymorphic data sets (with a minor allele frequency $<0.01 \%$ for autosomal dominant and $<0.1 \%$ for autosomal recessive genes) with less than 1 occurrence in homozygosity in 
gnomADv2.1 (gnomad.broadinstitute.org/; macarthurlab. org/2018/10/17/gnomad-v2-1). As reported previously, we determined predictably or probably deleterious scores using an in silico pipeline using a set of 10 prediction software packages. ${ }^{8}$ Putatively deleterious variants were validated by PCR-based standard capillary Sanger sequencing, both in patients and in relatives whose DNA was available for segregation studies, also to determine inheritance and phases of multiple gene variants and to establish whether variants had occurred de novo. Segregation in affected and unaffected relatives made it possible to better define pathogenic variants once we had identified those more likely to be disease causative.

Routine morphology and immunofluorescence analysis of muscle proteins were performed on vastus lateralis biopsy samples according to standard protocols.

\section{Data availability}

The data set used and analyzed during the current study is available from the corresponding author on reasonable request.

\section{Results}

We evaluated 66 patients with hyperCKemia (44 men and 22 women, age range 5-73 years), referred over an approximately 2 -year period to tertiary neuromuscular centers.
Most of the patients were Italian, adults ( $\geq 16$ years), and apparently sporadic cases. Although none of them had obvious neuromuscular disorders, 16 had a positive family history of muscle weakness or fatigue compatible with autosomal dominant inheritance in 13 and a recessive pattern of inheritance in 3. Their mean age at onset (i.e., at the time of the first documented evidence of hyperCKemia) was $26.7 \pm 19.2$ years, when they had serum CK levels ranging from 300 to $103,000 \mathrm{UI} / \mathrm{L}$ (normal $<190 \mathrm{UI} / \mathrm{L}$ ). Their disease duration ranged from 3 months to 30 years. Clinical examination revealed mild limbgirdle muscle weakness (median Medical Research Council scale score of 5 in upper girdle and $4+$ in lower girdle muscle) in 19 patients (figures 1-2). The distribution of the muscle weakness in these symptomatic patients is presented in figure 2 using the Human Phenotype Ontology (HPO) ID codes and nomenclature. ${ }^{9}$ Thirteen participants reported occasional exercise intolerance and myalgia, but showed no clear evidence of muscle weakness. Thirty-four patients remained completely asymptomatic over a 2-year observational period (figure 1). The patients' serum CK levels at their latest neurologic examination ranged from 150 to $64,610 \mathrm{UI} / \mathrm{L}$.

Cardiac examination, performed at some point during the course of the clinical follow-up, was unremarkable in all the patients except 4 whose ECG showed arrhythmias of uncertain significance. Pulmonary and functional respiratory tests were within normal limits in all the patients and remained so throughout the study.

Figure 1 Flowchart of patients' enrollment and results of genetic testing

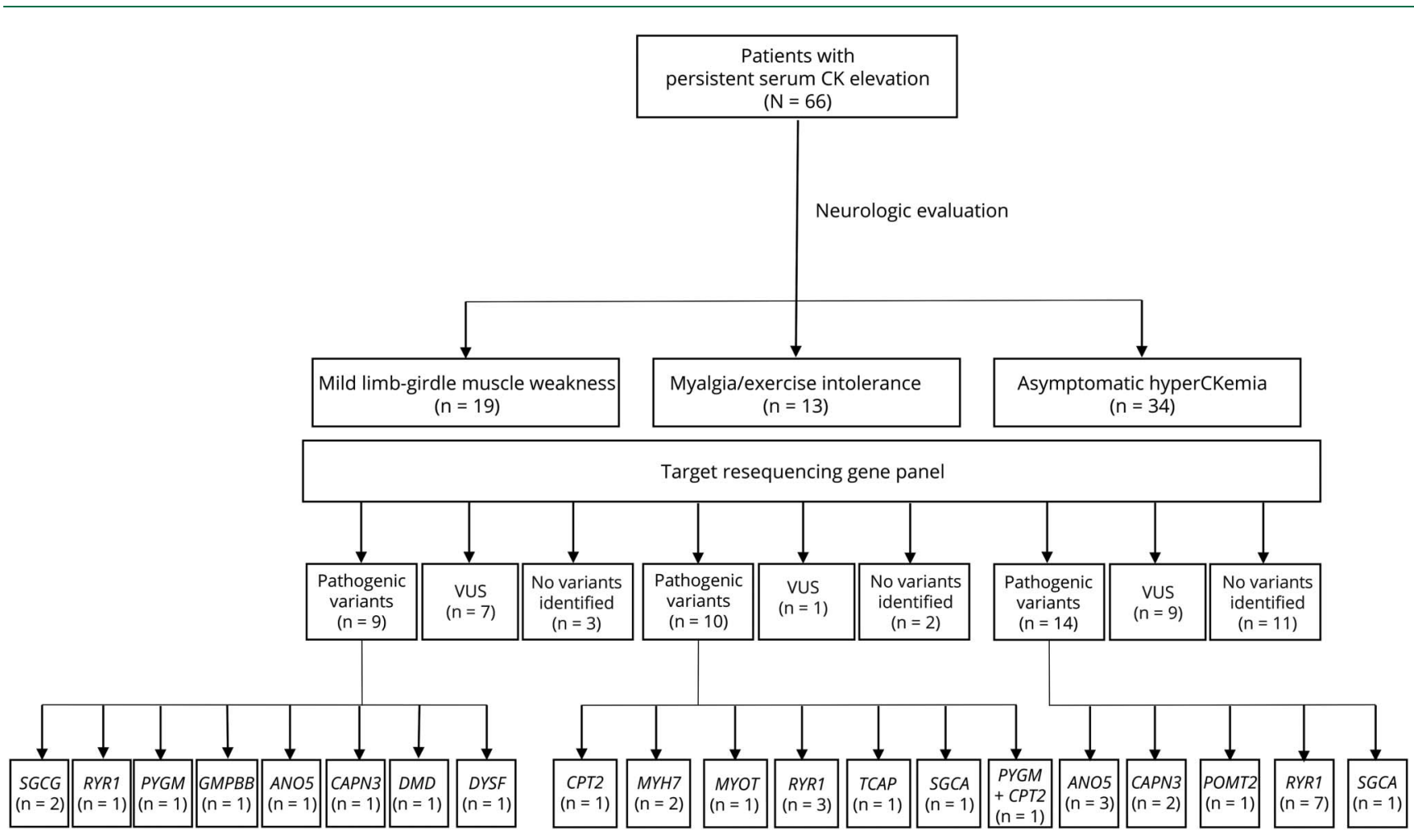

VUS = variants of unknown significance. 
EMG, performed on average 1 year after hyperCKemia onset, showed myopathic changes in 17 patients. Few patients underwent repeated neurophysiologic follow-up, and none of these showed substantial modifications. Twenty-six patients underwent muscle MRI scans at least once during the study, and these scans were unremarkable in 17 and not informative in the other 9. Fifty-seven patients underwent a skeletal muscle biopsy. In 8 cases (14\%), this showed clear dystrophic changes, with fat and connective tissue replacement suggesting a possible diagnosis of muscular dystrophy, whereas the vast majority $(38 / 57,67 \%)$ showed only mild, nonspecific myopathic changes, with normal muscle protein staining and no abnormal metabolic features (figure 3 and supplementary figure e-1, links.lww.com/NXG/A175). Muscle biopsy was normal in 11 patients.

Through multigene targeted NGS analysis, we identified an average of 50 rare variants in each patient. To facilitate our "needle in a haystack" search, we used a stringent set of bioinformatics filters, including effects on the protein, scores for predicting pathogenicity, allele frequencies in public and internal databases, and annotation as disease-associated variants with Alissa, a customizable pipeline tool. ${ }^{10}$ Presence of the specific variant in the Human Genome Mutation Database (hgmd.cf.ac.uk) was not considered a mandatory criterion for attributing pathogenic significance. Bioinformatics data were combined and critically reevaluated taking into consideration clinical presentation with the relative HPO definition, age at onset, and segregation studies in familial cases. Moreover, in cases submitted to muscle biopsy, we integrated histologic and immunofluorescence features.

On the basis of these procedural steps and criteria, already used by us in previous research, ${ }^{7}$ we assigned a confirmatory genetic diagnosis to 19 patients presenting pathogenic, disease-associated $(n=13)$, or likely pathogenic $(n=6)$ variants. We also identified 14 patients harboring changes predicted to affect function in genes corresponding to the clinical suspicions in these cases, thereby increasing group of individuals with a molecular diagnosis to 33 patients. We confirmed the molecular diagnosis using segregation analysis or immunofluorescence, or Western blotting on muscle

Figure 2 Clinical features of symptomatic patients presented using HPO ID codes and nomenclature

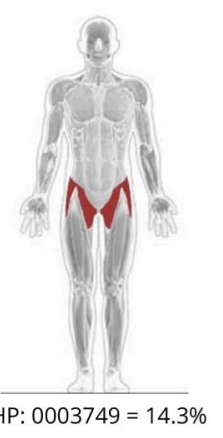

HP: $0003749=14.3 \%$
A

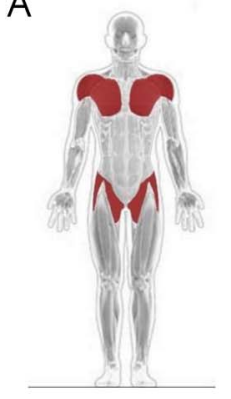

$\mathrm{HP}: 0003325=37.1 \%$

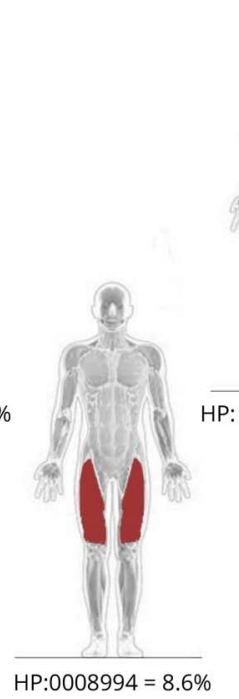

B. Symptomatic patients
(A) Muscle weakness pattern frequencies expressed as percentage rates. Clinical phenotypes are indicated using the HPO ID codes. HP:0003325 = limb-girdle muscle weakness; HP:0008994 = proximal muscle weakness in lower limbs; HP:0003749 = pelvic girdle muscle weakness. (B) Frequencies of clinical phenotypes associated with hyperCKemia expressed as percentage rates. Clinical phenotypes are indicated using HPO codes and nomenclature. HPO = human phenotype ontology. 
Figure 3 Myopathologic changes in patients with limb-girdle muscle weakness (top row, A-C), myalgia, and exercise intolerance (middle row, D-F) and in asymptomatic patients (bottom row, G-I)

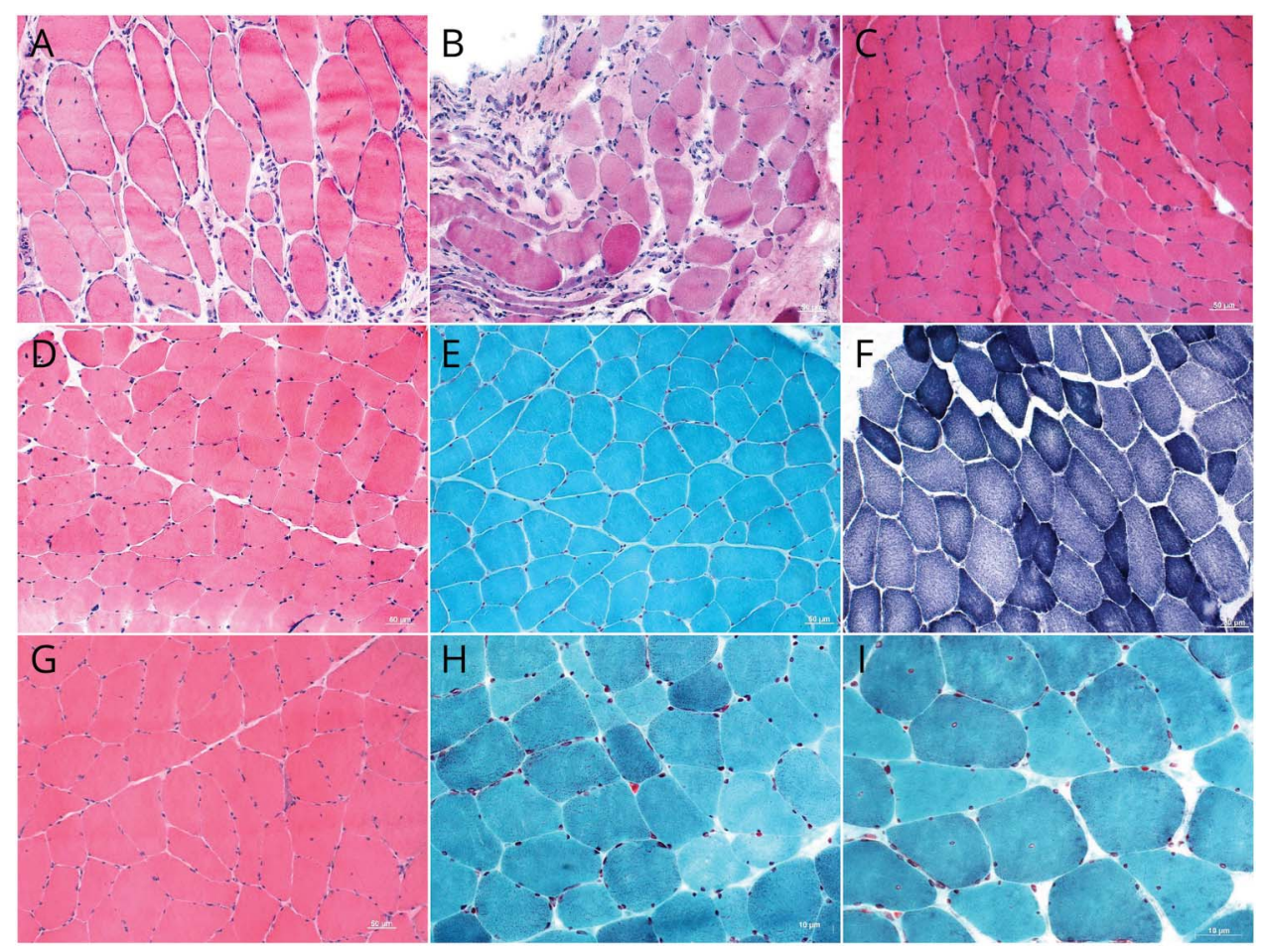

(A and B) Hematoxylin and eosin (HE) staining demonstrating marked variation in fiber size, several central nuclei, and increased connective tissue staining in P4 and P16, respectively. (C) $\mathrm{HE}$ staining showing several degenerating fibers in P29. (D) $\mathrm{HE}$ staining revealing slight variation in fiber size and some central nuclei in P3. (E) Gomori trichrome staining showing some hypotrophic fibers and increased internal nuclei in P28. (F) $\mathrm{NADH}-\mathrm{TR}$ staining showing motheaten fibers in P33. (G) HE staining demonstrating mild variation in fiber size and a few central nuclei in P7. $(H$ and I) Gomori trichrome staining showing slight variation in fiber size and some internal nuclei in P19 and $\mathrm{P} 22$, respectively. Magnification 200×.

biopsies, or their combination. Detailed information on these patients, including clinical characteristics, CK levels, muscle biopsy, and gene mutations, is shown in tables 1 and 2, whereas the genetic data are summarized in figure 4 and further detailed in supplementary web data (tables e-1 to e-4, links.lww.com/ NXG/A176, links.lww.com/NXG/A177, links.lww.com/ NXG/A178, and links.lww.com/NXG/A179).

Of the aforementioned 33 patients, 9 showed mild limb-girdle muscle weakness, 10 showed myalgia and/or occasional exercise intolerance, and 1 case rhabdomyolysis, whereas 14 were asymptomatic despite having muscle biopsies showing low immunofluorescence for specific proteins (Figure 1, figure e-1, links.lww.com/NXG/A175). Immunofluorescence analysis was performed to corroborate loss-of-function variants in 7 cases. With regard to the genetic analyses, pathogenic variants in RYR1 were found in 11 patients, in ANOS in 4. Four patients harbored pathogenic variants in genes encoding sarcoglycans, whereas 3 in CAPN3 (figure 4). Serum CK levels in the group of molecularly defined patients ranged from 600 to $67,490 \mathrm{UI} / \mathrm{L}$, whereas muscle biopsy (performed in $31 / 33$ individuals) showed evidence of dystrophic processes in 6 (figure 3, figure e-1), mild nonspecific muscle changes in 21 , and no noteworthy alterations in 4 patients.

Variants of unknown significance (VUS) with a potential causative role were found in 17 additional patients (supplementary table e-3, links.lww.com/NXG/A178). In these cases, either there was only a partial correspondence with the clinical phenotype or we discovered a single truncating variant (or a known disease-associated variant) in a recessive candidate gene. Most VUS occurred in 3 genes, namely CAPN3, CPT2, and ANO5 (figure 1 and supplementary table e-3, links.lww.com/NXG/ A178). Although it is possible that the low number of genes in our panel limited its informativeness on the patients' genotypes, it is also conceivable that in the VUS subgroup, the second allele may carry a deep intronic change leading to a mRNA splicing defect or large scale gene deletions/duplications, or a variation in genomic regulatory regions not covered in our study. Our study explored none of these possibilities.

Sixteen patients harbored molecular findings not compatible with their phenotypes. Although we cannot exclude a possible role for some of the variants detected in this subgroup, other additional and undetected genetic changes could well be responsible for their phenotypes.

\section{Discussion}

Both the EFNS guidelines ${ }^{1}$ and the authors of a more recent review article $^{35}$ recommend conducting further investigations in individuals with apparently idiopathic hyperCKemia if the CK level is repeatedly more than 3 times higher than the ULN, EMG reveals myopathic changes, and the patient is younger than 25 years. However, it is anticipated that advancing knowledge in the 
Table 1 Clinical and genetic features in 13 patients with pathogenic variants

\begin{tabular}{|c|c|c|c|c|c|c|c|c|c|c|}
\hline ID & $\begin{array}{l}\text { Sex/ } \\
\text { age }(y)\end{array}$ & $\begin{array}{l}\text { Serum CK } \\
\text { (UI/L) }\end{array}$ & Clinical findings & HPO ID code & $\begin{array}{l}\text { Muscle } \\
\text { biopsy }\end{array}$ & $\begin{array}{l}\text { Mutated } \\
\text { gene }\end{array}$ & Mutation cDNA level & Mutation protein level & Inheritance & Reference \\
\hline P9 & $\mathrm{F} / 54 \mathrm{y}$ & 1,310 & Asymptomatic & NA & NA & $\mathrm{ANOS}^{\mathrm{b}}$ & c. $1733 T>C / c .2295 C>G$ & p.Phe578Ser/p.Tyr765* & $A R$ & [11] \\
\hline P10 & F/61 y & 2000 & Limb-girdle muscle weakness & HP:0003325 & $\begin{array}{l}\text { Mild } \\
\text { myopathic } \\
\text { signs }\end{array}$ & $C A P N 3^{\mathrm{b}}$ & $\begin{array}{l}\text { c.633G }>\text { T/ } \\
\text { c.1537insCCCCATCTCTCAG }\end{array}$ & $\begin{array}{l}\text { p.Lys211Asn/ } \\
\text { Met513Thrfs*68 }\end{array}$ & $\mathrm{AR}$ & [12] \\
\hline P13 & $M / 32 y$ & 1800 & Myalgia and exercise intolerance & $\begin{array}{l}\text { HP:0003326; HP: } \\
0009020\end{array}$ & Normal & $T C A P^{C}$ & c.33_35del/- & p.Glu12del/- & $A D$ & This work \\
\hline P15 & $M / 7 y$ & 7,348 & Asymptomatic & NA & NA & CAPN $3^{\mathrm{b}}$ & c.549delA/c.2115+2T>A & $\begin{array}{l}\text { p.Thr184Argfs*35/splice } \\
\text { site }^{a}\end{array}$ & $A R$ & {$[13,14]$} \\
\hline P16 & $M / 14 y$ & 2,800 & Limb-girdle muscle weakness & HP:0003325 & $\begin{array}{l}\text { Dystrophic } \\
\text { signs }\end{array}$ & $S G C G^{c}$ & c. $195+1 G>A / c .195+1 G>A$ & Splice site ${ }^{a} /$ splice site ${ }^{a}$ & $A R$ & This work \\
\hline P22 & $F / 48$ y & 2000 & Asymptomatic & NA & $\begin{array}{l}\text { Mild } \\
\text { myopathic } \\
\text { signs }\end{array}$ & $A N O 5^{\mathrm{b}}$ & c. $1356 C>G / c .1356 C>G$ & p.Tyr452*/p.Tyr452* & $\mathrm{AR}$ & This work \\
\hline P25 & $\mathrm{M} / 11 \mathrm{y}$ & 19,000 & $\begin{array}{l}\text { Exercise intolerance and pelvic girdle } \\
\text { muscle weakness }\end{array}$ & $\begin{array}{l}\text { HP:0009020; HP: } \\
0003749\end{array}$ & $\begin{array}{l}\text { Dystrophic } \\
\text { signs }\end{array}$ & $D M D^{C}$ & c.151_153del & p.Leu51del & $X L$ & This work \\
\hline P27 & F/67 y & 900 & Pelvic girdle muscle weakness & HP:0003749 & $\begin{array}{l}\text { Mild } \\
\text { myopathic } \\
\text { signs }\end{array}$ & PYGM ${ }^{c}$ & c. $2262 \mathrm{del} A / c .406 \mathrm{G}>\mathrm{A}$ & $\begin{array}{l}\text { p.Lys754Asnfs*49/ } \\
\text { p.Gly136Ser }\end{array}$ & $\mathrm{AR}$ & [14] \\
\hline P28 & $F / 32$ y & 4500 & Exercise intolerance and myalgia & $\begin{array}{l}\text { HP:0009020; HP: } \\
0003326\end{array}$ & $\begin{array}{l}\text { Mild } \\
\text { myopathic } \\
\text { signs }\end{array}$ & $S G C A^{C}$ & c. $739 \mathrm{G}>\mathrm{A} / \mathrm{c} .850 \mathrm{C}>\mathrm{T}$ & p.Val247Met/p.Arg284Cys & AR & {$[15,16]$} \\
\hline P29 & $\mathrm{F} / 6 \mathrm{y}$ & 16,340 & Limb-girdle muscle weakness & HP:0003325 & $\begin{array}{l}\text { Dystrophic } \\
\text { signs }\end{array}$ & $S G C G^{c}$ & c.521 delT/c.521 delT & $\begin{array}{l}\text { p.Leu174Leufs } * 21 / \\
\text { p.Leu174Leufs } * 21\end{array}$ & $A R$ & This work \\
\hline P30 & $M / 66 y$ & 1800 & Asymptomatic & NA & $\begin{array}{l}\text { Mild } \\
\text { myopathic } \\
\text { signs }\end{array}$ & $A N O 5^{\mathrm{b}}$ & c.629C>T/c.726dupT & $\begin{array}{l}\text { p.Ser210Leu/ } \\
\text { p.Ser243Phefs*2 }\end{array}$ & AR & [17] \\
\hline P32 & $M / 53$ y & 780 & Myalgia and exercise intolerance & $\begin{array}{l}\text { HP:0003326; HP: } \\
0009020\end{array}$ & $\begin{array}{l}\text { Mild } \\
\text { myopathic } \\
\text { signs }\end{array}$ & $\begin{array}{l}\text { PYGM/ } \\
\text { CPT2 }\end{array}$ & $\begin{array}{l}\text { PYGM: c. }[148 \mathrm{C}>\mathrm{T}] ; \text {; } C P T 2: \\
\text { c.[1348A>T] }\end{array}$ & $\begin{array}{l}\text { PYGM:p.[Arg50*]; CPT2: } \\
\text { p.[Arg450*] }\end{array}$ & NA & [18-20] \\
\hline P33 & $\mathrm{M} / 18$ & 2,937 & Myalgia and exercise intolerance & $\begin{array}{l}\text { HP:0003326; HP: } \\
0009020\end{array}$ & $\begin{array}{l}\text { Mild myopathic } \\
\text { signs }\end{array}$ & $M Y H 7^{\mathrm{b}}$ & c.3790delG/- & p.Glu1264Argfs*34/- & $A D$ & This work \\
\hline
\end{tabular}

Abbreviations: $A D=$ autosomal dominant; $A R=$ autosomal recessive; $H P O=$ Human Phenotype Ontology; $N A=$ not available; $X L=X$-linked

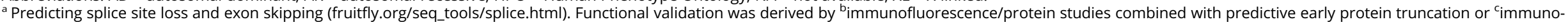
fluorescence studies only. 
Table 2 Clinical and genetic features in 20 patients with "likely" pathogenic variants

\begin{tabular}{|c|c|c|c|c|c|c|c|c|c|c|}
\hline ID & $\begin{array}{l}\text { Sex } / \\
\text { age } \\
\text { (y) }\end{array}$ & $\begin{array}{l}\text { Serum } \\
\text { CK (UII } \\
\text { L) }\end{array}$ & $\begin{array}{l}\text { Clinical } \\
\text { findings }\end{array}$ & $\begin{array}{l}\text { HPO ID } \\
\text { code }\end{array}$ & $\begin{array}{l}\text { Muscle } \\
\text { biopsy }\end{array}$ & $\begin{array}{l}\text { Mutated } \\
\text { gene }\end{array}$ & $\begin{array}{l}\text { Mutation } \\
\text { cDNA level }\end{array}$ & $\begin{array}{l}\text { Mutation } \\
\text { protein level }\end{array}$ & Inheritance & Reference \\
\hline P1 & $\begin{array}{l}M / \\
19 y\end{array}$ & 3,000 & Asymptomatic & NA & $\begin{array}{l}\text { Mild } \\
\text { myopathic } \\
\text { signs }\end{array}$ & RYR1 & c. $8888 \mathrm{~T}>\mathrm{C} /-$ & p.Leu2963Pro/- & $A D$ & [21] \\
\hline P2 & $\begin{array}{l}M / \\
56 y\end{array}$ & 800 & Asymptomatic & NA & $\begin{array}{l}\text { Mild } \\
\text { myopathic } \\
\text { signs }\end{array}$ & $R Y R 1^{\mathrm{b}}$ & c. $7373 \mathrm{G}>\mathrm{A} /-$ & p.Arg2458His/- & $A D$ & [22] \\
\hline P3 & $\begin{array}{l}M / \\
68 y\end{array}$ & 816 & $\begin{array}{l}\text { Exercise } \\
\text { intolerance }\end{array}$ & $\begin{array}{l}\text { HP: } \\
0009020\end{array}$ & $\begin{array}{l}\text { Mild } \\
\text { myopathic } \\
\text { signs }\end{array}$ & RYR1 & c.1163C>T/- & p.Ser388Leu/- & NA & This work \\
\hline $\mathrm{P4}^{\mathrm{d}}$ & $\begin{array}{l}M / \\
48 y\end{array}$ & 1,044 & $\begin{array}{l}\text { Limb-girdle } \\
\text { muscle } \\
\text { weakness }\end{array}$ & $\begin{array}{l}\text { HP: } \\
0003325\end{array}$ & $\begin{array}{l}\text { Dystrophic } \\
\text { signs }\end{array}$ & $G M P P B^{\mathrm{b}}$ & $\begin{array}{l}\text { c. } 95 \mathrm{C}>\mathrm{T} / \\
\text { c. } 727 \mathrm{C}>\mathrm{T}\end{array}$ & $\begin{array}{l}\text { p.Pro32Leu/ } \\
\text { p.Arg243Trp }\end{array}$ & AR & {$[8,23]$} \\
\hline P5 & $\begin{array}{l}F / 65 \\
y\end{array}$ & 600 & Myalgia & $\begin{array}{l}\text { HP: } \\
0003326\end{array}$ & $\begin{array}{l}\text { Mild } \\
\text { myopathic } \\
\text { signs }\end{array}$ & MYH7 & c.2009T>C/- & p.Val670Ala/- & NA & This work \\
\hline P6 & $\begin{array}{l}M / \\
17 y\end{array}$ & 865 & Asymptomatic & NA & $\begin{array}{l}\text { Mild } \\
\text { myopathic } \\
\text { signs }\end{array}$ & $R Y R 1^{\mathrm{b}}$ & c.7291G>A/- & p.Asp2431Asn/- & NA & [24] \\
\hline P7 & $\begin{array}{l}M / \\
32 y\end{array}$ & 1,000 & Asymptomatic & NA & $\begin{array}{l}\text { Mild } \\
\text { myopathic } \\
\text { signs }\end{array}$ & $R Y R 1^{\mathrm{b}}$ & c.5036G>A/- & p.Arg1679His/- & $A D$ & [25] \\
\hline P8 & $\begin{array}{l}M / \\
40 y\end{array}$ & 1,000 & Myalgia & $\begin{array}{l}\text { HP: } \\
0003326\end{array}$ & $\begin{array}{l}\text { Mild } \\
\text { myopathic } \\
\text { signs }\end{array}$ & $R Y R 1^{\mathrm{b}}$ & c.7048G>A/- & p.Ala2350Thr/- & $A D$ & [26] \\
\hline P11 & $\begin{array}{l}F / 51 \\
y\end{array}$ & 3,000 & $\begin{array}{l}\text { Limb-girdle } \\
\text { muscle } \\
\text { weakness }\end{array}$ & $\begin{array}{l}\text { HP: } \\
0003325\end{array}$ & $\begin{array}{l}\text { Dystrophic } \\
\text { signs }\end{array}$ & $D Y S F^{c}$ & $\begin{array}{l}\text { c. } 862 \mathrm{G}>\mathrm{T} / \\
\text { c. } 2875 \mathrm{C}>\mathrm{T}\end{array}$ & $\begin{array}{l}\text { p.Asp288Tyr/ } \\
\text { p.Arg959Trp }\end{array}$ & $A R$ & [27] \\
\hline P12 & $\begin{array}{l}M / \\
11 y\end{array}$ & 728 & $\begin{array}{l}\text { Myalgia and } \\
\text { exercise } \\
\text { intolerance }\end{array}$ & $\begin{array}{l}\text { HP: } \\
\text { 0003326; } \\
\text { HP: } \\
0009020\end{array}$ & $\begin{array}{l}\text { Mild } \\
\text { myopathic } \\
\text { signs }\end{array}$ & $R Y R 1^{\mathrm{b}}$ & c.6599C>T/- & p.Ala2200Val/- & $A D$ & [28] \\
\hline P14 & $\begin{array}{l}F / 65 \\
y\end{array}$ & 3,300 & $\begin{array}{l}\text { Limb-girdle } \\
\text { muscle } \\
\text { weakness }\end{array}$ & $\begin{array}{l}\text { HP: } \\
0003325\end{array}$ & $\begin{array}{l}\text { Dystrophic } \\
\text { signs }\end{array}$ & ANO5 & $\begin{array}{l}\text { c. } 580 \mathrm{C}>\mathrm{T} / \\
\text { c. } 2219 \mathrm{C}>\mathrm{T}\end{array}$ & $\begin{array}{l}\text { p.Arg194Trp/ } \\
\text { p.Ser740Phe }\end{array}$ & $A R$ & This work \\
\hline P17 & $\begin{array}{l}\mathrm{M} / 5 \\
\mathrm{y}\end{array}$ & 67,490 & Rhabdomyolysis & $\begin{array}{l}\text { HP: } \\
0003201\end{array}$ & $\begin{array}{l}\text { Mild } \\
\text { myopathic } \\
\text { signs }\end{array}$ & $C P T 2^{\mathrm{b}}$ & $\begin{array}{l}\text { c. } 338 \mathrm{C}>\mathrm{T} / \\
\text { c. } 338 \mathrm{C}>\mathrm{T}\end{array}$ & $\begin{array}{l}\text { p.Ser113Leu/ } \\
\text { p.Ser113Leu }\end{array}$ & $A R$ & [29] \\
\hline P18 & $\begin{array}{l}M / \\
29 y\end{array}$ & 600 & Asymptomatic & NA & Normal & $R Y R 1^{\mathrm{b}}$ & $\begin{array}{l}\text { c. }[4711 \mathrm{~A}>\mathrm{G} ; \\
10097 \mathrm{G}>\mathrm{A}] / \\
\text { c. } 11798 \mathrm{~A}>\mathrm{G}\end{array}$ & $\begin{array}{l}\text { p.[(Ile1571Val; } \\
\text { Arg3366His)]/ } \\
\text { p.Tyr3933Cys }\end{array}$ & NA & [30-32] \\
\hline P19 & $\begin{array}{l}M / \\
39 y\end{array}$ & 2000 & Asymptomatic & NA & Normal & CAPN3 & $\begin{array}{l}\text { c. }[1,395 \\
1397 \mathrm{del} ; \\
2257 \mathrm{G}>\mathrm{A}] / \\
1453 \mathrm{~A}>\mathrm{G}\end{array}$ & $\begin{array}{l}\text { p.[(Leu465_- } \\
\text { Glu466del; } \\
\text { Asp753Asn)]/ } \\
\text { p.Met485Val }\end{array}$ & $\mathrm{AR}$ & {$[12,32,33]$} \\
\hline $\mathrm{P}^{2} \mathrm{O}^{\mathrm{d}}$ & $\begin{array}{l}M / \\
24 y\end{array}$ & 12,000 & Asymptomatic & NA & $\begin{array}{l}\text { Mild } \\
\text { myopathic } \\
\text { signs }\end{array}$ & $S G C A$ & $\begin{array}{l}\text { c. } 242 \mathrm{G}>\mathrm{A} / \\
\text { c. } 739 \mathrm{G}>\mathrm{A}\end{array}$ & $\begin{array}{l}\text { p.Arg81His/ } \\
\text { p.Val247Met }\end{array}$ & NA & [15] \\
\hline P21 & $\begin{array}{l}F / 53 \\
y\end{array}$ & 500 & $\begin{array}{l}\text { Limb-girdle } \\
\text { muscle } \\
\text { weakness }\end{array}$ & $\begin{array}{l}\text { HP: } \\
0003325\end{array}$ & $\begin{array}{l}\text { Mild } \\
\text { myopathic } \\
\text { signs }\end{array}$ & RYR1 & $\begin{array}{l}\text { c.10923- } \\
\text { 8C>A/- }\end{array}$ & Splice site ${ }^{a} /-$ & NA & This work \\
\hline P23 & $\begin{array}{l}M / \\
64 \text { y }\end{array}$ & 1,000 & Asymptomatic & NA & $\begin{array}{l}\text { Mild } \\
\text { myopathic } \\
\text { signs }\end{array}$ & RYR1 & c. $14812 A>G /-$ & p.lle4938Val/- & NA & This work \\
\hline$P 24^{d}$ & $\begin{array}{l}M / \\
67 y\end{array}$ & 1,103 & Myalgia & $\begin{array}{l}\text { HP: } \\
0003326\end{array}$ & $\begin{array}{l}\text { Mild } \\
\text { myopathic } \\
\text { signs }\end{array}$ & MYOT & c. $179 \mathrm{C}>\mathrm{T} /-$ & p.Ser60Phe/- & $A D$ & [34] \\
\hline
\end{tabular}


Table 2 Clinical and genetic features in 20 patients with "likely" pathogenic variants (continued)

\begin{tabular}{|c|c|c|c|c|c|c|c|c|c|c|}
\hline ID & $\begin{array}{l}\text { Sex/ } \\
\text { age } \\
\text { (y) }\end{array}$ & $\begin{array}{l}\text { Serum } \\
\text { CK (UI/ } \\
\text { L) }\end{array}$ & $\begin{array}{l}\text { Clinical } \\
\text { findings }\end{array}$ & $\begin{array}{l}\text { HPO ID } \\
\text { code }\end{array}$ & $\begin{array}{l}\text { Muscle } \\
\text { biopsy }\end{array}$ & $\begin{array}{l}\text { Mutated } \\
\text { gene }\end{array}$ & $\begin{array}{l}\text { Mutation } \\
\text { cDNA level }\end{array}$ & $\begin{array}{l}\text { Mutation } \\
\text { protein level }\end{array}$ & Inheritance & Reference \\
\hline$P 26^{d}$ & $\begin{array}{l}\mathrm{M} / \\
21 \mathrm{y}\end{array}$ & 1929 & Asymptomatic & NA & Normal & POMT2 ${ }^{\mathrm{C}}$ & $\begin{array}{l}\text { c. }[1733 \mathrm{G}>\mathrm{A} ; \\
239 \mathrm{C}>\mathrm{T}] / \\
\text { c. } 707 \mathrm{~T}>\mathrm{G}\end{array}$ & $\begin{array}{l}\text { p.[(Arg578His; } \\
\text { Pro80Leu)]/ } \\
\text { p.Leu236Arg }\end{array}$ & AR & This work \\
\hline P31 & $\begin{array}{l}M / \\
26 y\end{array}$ & 40,000 & Asymptomatic & NA & $\begin{array}{l}\text { Mild } \\
\text { myopathic } \\
\text { signs }\end{array}$ & $R Y R 1^{\mathrm{C}}$ & $\begin{array}{l}\text { c. } 5288 C>T / \\
\text { c. } 7681 C>T\end{array}$ & $\begin{array}{l}\text { p.Pro1763Leu/ } \\
\text { p.Leu2561Phe }\end{array}$ & NA & This work \\
\hline
\end{tabular}

Abbreviations: $\mathrm{AD}=$ autosomal dominant; $\mathrm{AR}=$ autosomal recessive; $\mathrm{HPO}=$ human phenotype ontology; $\mathrm{NA}=$ not available.

apredicting splice site loss and exon skipping (fruitfly.org/seq_tools/splice.html).

${ }^{b}$ Functional validation derived by immunofluorescence/protein studies combined with predictive early protein truncation/previous published work with evidences of pathogenicity.

${ }^{c}$ Functional validation derived through immunofluorescence studies only.

"Patients with "likely" pathogenic variants confirmed by immunofluorescence analysis or previously published evidence of pathogenicity.

field of muscle disorders will lead to the identification of previously unrecognized causes of hyperCKemia, thereby reducing the proportion of idiopathic cases. This would be an important development, as it would allow more precise clinical monitoring and follow-up and help to clarify the natural history of the different forms.

Studies conducted before NGS entered the clinical arena considered the diagnostic impact of muscle biopsy in the evaluation of patients with asymptomatic hyperCKemia ${ }^{2,3,36-38}$ and the relative frequency of common genes such as $D M D .{ }^{38}$ In recent years,
NGS, which has involved the development of different gene panels and seen the focus switch to peculiar muscle phenotypes, has transformed the approach to the study of neuromuscular disorders (the relevant data are for the most part reviewed in reference 4). Some studies have also addressed, among other aspects, the genotype of patients with isolated hyperCKemia, reporting a limited diagnostic yield (21\%) in fewer than 40 patients. 39,40

Herein, we report the results obtained in a cohort of 66 patients with hyperCKemia, half of whom were asymptomatic on evaluation. Our use of massive parallel sequencing with

Figure 4 Diagnostic rates and molecular results

A

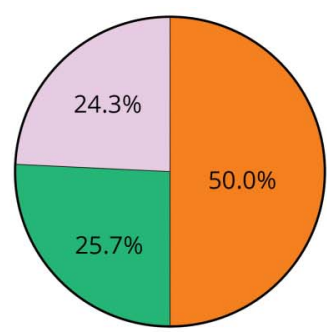

Variants

- Pathogenic or likely pathogenic

- Unknown significance

No putative causative

B

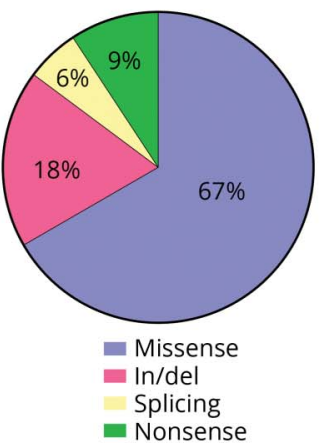

C

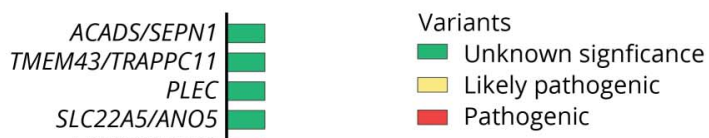

LDB3/DNAJB6

POMT2

MYOT

CPT2
DYSF

GMPPB

RYR1

$M Y H 7$

PYGM/CPT

SGCA

PYGM

$D M D$

SGCG

TCAP

CAPN3
ANO5

Number of patients

(A) Pathogenic or likely pathogenic variants were found in $50 \%$ of the patients, whereas $25.7 \%$ showed variants that required further characterization, and $24.3 \%$ did not present pathogenic variants. (B) Type of causative mutations identified in diagnosed patients: missense (67\%), small indels (18\%), splice site variants (6\%), and nonsense mutations (9\%). (C) Bar chart showing number of patients with "pathogenic" (red), "likely pathogenic" variants (yellow), and variants of unknown significance (green) for each gene. 
a multigene panel combined with the application of stringent bioinformatics filters, and integration of muscle phenotypes (both clinical and morphological), produced a 50\% diagnostic yield, a rate higher than those previously reported. ${ }^{39,40}$ This difference is likely related to the combination of genotype with integrated clinico-morphological phenotype data in the present study, to its more homogeneous population (mostly adults in our cohort as opposed to the mixed populations studied by others), to technical improvements implicit in more recent gene panel technologies, or to a combination of these factors. There were no straightforward differences between the solved and unsolved participants, with clinical features and serum CK values being roughly the same in the 2 subgroups. Our data confirm that main challenges in NGS data analysis are the clinical interpretation of molecular findings and the distinction of causative mutations from the plethora of not clinically significant DNA variations. The low frequency of variants, or their absence in reference polymorphic databases, is recognized as a necessary but not sufficient criterion to assign pathogenicity. Moreover, in silico predictions present often unanticipated discordance between different predicting algorithms. ${ }^{4}$ All this make more complex to reach a final molecular diagnosis. Considering our results overall, there emerge several points worth underlining. First, of the 33 patients who received a molecular diagnosis, 11 harbored mutations in RYR1, and all of these presented normal muscle MRI scans and subtle muscle involvement on skeletal muscle biopsy. Only 1 presented episodes of rhabdomyolysis, 2 presented myalgia, and 1 showed mild limb-girdle muscle weakness. Conversely, 7 patients were fully asymptomatic with a normal clinical evaluation associated with serum steady CK levels ranging from 500 to $1,000 \mathrm{UI} / \mathrm{L}$. Although we did not seek to further corroborate our genomic findings by evaluating their functional impact on intracellular calcium homeostasis through complex studies or by performing immunoblotting with commercial antibodies, all the detected RYR 1 variants were rare ones, namely those inherited segregated in familial cases with clinical manifestations, and all are likely to perturb secondary ryanodine-1 receptor structure in protein modeling (not shown). Overall, our data imply that mutations in RYR1 may be a common in hyperCKemia, even when CK levels are $<1,000 \mathrm{UI} / \mathrm{L}$. Of interest, we observed that the RYR1 participants presented pseudometabolic features more frequently than patients harboring other genotypes but did not stand up as a clinically different subgroup. Second, we identified biallelic mutations in ANOS in 4 patients and mutations in SGCA in 2 adults with long-lasting hyperCKemia without muscle weakness. These data support previous impressions suggesting that analysis of known LGMD genes is justified even when isolated hyperCKemia or minimal muscle weakness is the sole clinical manifestation. ${ }^{41,42}$ Third, we frequently observed mild, nonspecific muscle abnormalities consisting of variation in fiber size, nuclear internalization, or these 2 features combined; these alterations were always unrelated to serum CK levels, age at onset, clinical features, disease duration, and mutated gene. Nevertheless, muscle biopsy remains an important part of the diagnostic process in hyperCKemia, as it can also fulfill a "functional" role assisting in the clarification of uncertain cases. This is illustrated by case P28 in our study where 2 changes in SGCA were substantiated by reduced $\alpha$-sarcoglycan labeling in skeletal muscle (figure e-1, links.lww.com/NXG/A175, A-B). Fourth, 26\% of the patients were found to harbor VUS. Nonetheless, we frequently identified single deleterious variants in CAPN3 and CPT2, 2 autosomal recessive genes where manifesting heterozygosity reportedly occurs. ${ }^{43-45}$ The existence, in hyperCKemia, of symptomatic carriers, various degrees of clinical severity, and large intrafamilial heterogeneity of the phenotype (ranging from asymptomatic to fatal) could be explained by factors other than the genotype. In the case of CPT2-related myopathy, for example, one might consider different degrees of exposure to triggering/environmental factors (e.g., temperature, nutrition, fitness level) capable of increasing dependency on fat oxidation and further impairing exercise performance. ${ }^{46}$ Taking these factors into consideration, as well as the possibility of synergistic heterozygosity ${ }^{34}$ (as in our case P32) and digenic inheritance, it is imperative to combine thorough expert clinical and myopathologic evaluation with NGS as a prelude to a higher diagnostic rate in patients with paucisymptomatic hyperCKemia. Finally, isolated hyperCKemia can be the sole feature alerting to a progressive muscular disorder requiring careful surveillance and may open new prospective in future therapeutic opportunities.

\section{Acknowledgment}

The authors thank Catherine J. Wrenn, who provided expert editorial assistance.

\section{Study funding}

Funding partially provided by Telethon Foundation grants GUP13004 to GA) and Regione Toscana FAS SALUTE 2014 (CUP 4042.16092014.066000060 to FMS).

\section{Disclosure}

Disclosures available: Neurology.org/NG.

\section{Publication history}

Received by Neurology: Genetics March 21, 2019. Accepted in final form June 21, 2019.

\begin{tabular}{|c|c|c|c|}
\hline Name & Location & Role & Contribution \\
\hline $\begin{array}{l}\text { Anna } \\
\text { Rubegni, MD }\end{array}$ & $\begin{array}{l}\text { IRCCS } \\
\text { Stella } \\
\text { Maris, Pisa }\end{array}$ & Author & $\begin{array}{l}\text { Designed and conceptualized } \\
\text { the study; analyzed the data; } \\
\text { and drafted the manuscript for } \\
\text { intellectual content }\end{array}$ \\
\hline $\begin{array}{l}\text { Alessandro } \\
\text { Malandrini, } \\
\text { MD }\end{array}$ & $\begin{array}{l}\text { University } \\
\text { of Siena }\end{array}$ & Author & $\begin{array}{l}\text { Major role in the acquisition of } \\
\text { data and drafted the } \\
\text { manuscript for intellectual } \\
\text { content }\end{array}$ \\
\hline $\begin{array}{l}\text { Claudia Dosi, } \\
\text { MD }\end{array}$ & $\begin{array}{l}\text { University } \\
\text { of Pisa }\end{array}$ & Author & $\begin{array}{l}\text { Acquisition, analysis, and } \\
\text { interpretation of data and } \\
\text { critical revision of the } \\
\text { manuscript }\end{array}$ \\
\hline $\begin{array}{l}\text { Guja Astrea, } \\
\text { MD }\end{array}$ & $\begin{array}{l}\text { IRCCS } \\
\text { Stella } \\
\text { Maris, Pisa }\end{array}$ & Author & $\begin{array}{l}\text { Acquisition, analysis, and } \\
\text { interpretation of data and } \\
\text { critical revision of the } \\
\text { manuscript }\end{array}$ \\
\hline
\end{tabular}


Appendix (continued)

\begin{tabular}{|c|c|c|c|c|c|c|c|}
\hline Name & Location & Role & Contribution & Name & Location & Role & Contribution \\
\hline $\begin{array}{l}\text { Jacopo } \\
\text { Baldacci, BS }\end{array}$ & $\begin{array}{l}\text { IRCCS } \\
\text { Stella } \\
\text { Maris, Pisa }\end{array}$ & Author & $\begin{array}{l}\text { Acquisition, analysis, and } \\
\text { interpretation of data and } \\
\text { critical revision of the } \\
\text { manuscript }\end{array}$ & $\begin{array}{l}\text { Gabriele } \\
\text { Siciliano, MD }\end{array}$ & $\begin{array}{l}\text { University } \\
\text { of Pisa }\end{array}$ & Author & $\begin{array}{l}\text { Acquisition, analysis, and } \\
\text { interpretation of data and } \\
\text { critical revision of the } \\
\text { manuscript }\end{array}$ \\
\hline $\begin{array}{l}\text { Carla } \\
\text { Battisti, MD }\end{array}$ & $\begin{array}{l}\text { University } \\
\text { of Siena }\end{array}$ & Author & $\begin{array}{l}\text { Acquisition, analysis, and } \\
\text { interpretation of data and } \\
\text { critical revision of the } \\
\text { manuscript }\end{array}$ & $\begin{array}{l}\text { Deborah } \\
\text { Tolomeo, } \\
\text { MD }\end{array}$ & $\begin{array}{l}\text { University } \\
\text { of Pisa }\end{array}$ & Author & $\begin{array}{l}\text { Acquisition, analysis, and } \\
\text { interpretation of data and } \\
\text { critical revision of the } \\
\text { manuscript }\end{array}$ \\
\hline $\begin{array}{l}\text { Giulia } \\
\text { Bertocci, BSc }\end{array}$ & $\begin{array}{l}\text { IRCCS } \\
\text { Stella } \\
\text { Maris, Pisa }\end{array}$ & Author & $\begin{array}{l}\text { Acquisition, analysis, and } \\
\text { interpretation of data and } \\
\text { critical revision of the } \\
\text { manuscript }\end{array}$ & $\begin{array}{l}\text { Paola Tonin, } \\
\text { MD }\end{array}$ & $\begin{array}{l}\text { University } \\
\text { of Verona }\end{array}$ & Author & $\begin{array}{l}\text { Acquisition, analysis, and } \\
\text { interpretation of data and } \\
\text { critical revision of the } \\
\text { manuscript }\end{array}$ \\
\hline $\begin{array}{l}\text { M. Alice } \\
\text { Donati, MD }\end{array}$ & $\begin{array}{l}\text { AOU } \\
\text { Meyer, } \\
\text { Florence }\end{array}$ & Author & $\begin{array}{l}\text { Acquisition, analysis, and } \\
\text { interpretation of data and } \\
\text { critical revision of the } \\
\text { manuscript }\end{array}$ & $\begin{array}{l}\text { Nila Volpi, } \\
\text { MD }\end{array}$ & $\begin{array}{l}\text { University } \\
\text { of Siena }\end{array}$ & Author & $\begin{array}{l}\text { Acquisition, analysis, and } \\
\text { interpretation of data and } \\
\text { critical revision of the } \\
\text { manuscript }\end{array}$ \\
\hline $\begin{array}{l}\text { M. Teresa } \\
\text { Dotti, MD }\end{array}$ & $\begin{array}{l}\text { University } \\
\text { of Siena }\end{array}$ & Author & $\begin{array}{l}\text { Acquisition, analysis, and } \\
\text { interpretation of data and } \\
\text { critical revision of the } \\
\text { manuscript }\end{array}$ & $\begin{array}{l}\text { Filippo M. } \\
\text { Santorelli, } \\
\text { MD }\end{array}$ & $\begin{array}{l}\text { IRCCS } \\
\text { Stella } \\
\text { Maris, Pisa }\end{array}$ & Author & $\begin{array}{l}\text { Interpreted the data; revised } \\
\text { the manuscript for intellectual } \\
\text { content; and provided funds for } \\
\text { the study }\end{array}$ \\
\hline $\begin{array}{l}\text { Antonio } \\
\text { Federico, MD }\end{array}$ & $\begin{array}{l}\text { University } \\
\text { of Siena }\end{array}$ & Author & $\begin{array}{l}\text { Acquisition, analysis, and } \\
\text { interpretation of data and } \\
\text { critical revision of the } \\
\text { manuscript }\end{array}$ & $\begin{array}{l}\text { Denise } \\
\text { Cassandrini, } \\
\text { PhD }\end{array}$ & $\begin{array}{l}\text { IRCCS } \\
\text { Stella } \\
\text { Maris, Pisa }\end{array}$ & Author & $\begin{array}{l}\text { Interpreted the data and } \\
\text { revised the manuscript for } \\
\text { intellectual content }\end{array}$ \\
\hline
\end{tabular}

Appendix (continued)

\section{References}

1. Kyriakides T, Angelini C, Schaefer J, et al. European Federation of Neurological Societies. EFNS guidelines on the diagnostic approach to pauci- or asymptomatic hyperCKemia. Eur J Neurol 2010;17:767-773.

2. Silvestri NJ, Wolfe GI. Asymptomatic/pauci-symptomatic creatine kinase elevations (hyperckemia). Muscle Nerve 2013;47:805-815.

3. Malandrini A, Orrico A, Gaudiano C, et al. Muscle biopsy and in vitro contracture test in subjects with idiopathic HyperCKemia. Anesthesiology 2008;109:625-628.

4. Nigro V, Savarese M. Next-generation sequencing approaches for the diagnosis of skeletal muscle disorders. Curr Opin Neurol 2016;29:621-627.

5. Astrea G, Petrucci A, Cassandrini D, et al. Myoimaging in the NGS era: the discovery of a novel mutation in MYH7 in a family with distal myopathy and core-like features-a case report. BMC Med Genet 2016;17:25.

6. Musumeci O, la Marca G, Spada M, et al. LOPED study: looking for an early diagnosis in a late-onset Pompe disease high-risk population. J Neurol Neurosurg Psychiatry 2016;87:5-11

7. Savarese M, Di Fruscio G, Mutarelli M, et al. MotorPlex provides accurate variant detection across large muscle genes both in single myopathic patients and in pools of DNA samples. Acta Neuropathol Commun 2014;2:100.

8. Astrea G, Romano A, Angelini C, et al. Broad phenotypic spectrum and genotypephenotype correlations in GMPPB-related dystroglycanopathies: an Italian crosssectional study. Orphanet J Rare Dis 2018;13:170.

9. Thompson R, Straub V. Limb-girdle muscular dystrophies-international collaborations for translational research. Nat Rev Neurol 2016;12:294-309.

10. D'Amore A, Tessa A, Casali C, et al. Next generation revolution in molecular diagnosi of hereditary spastic paraplegias: a large Italian cross-sectional study. Front Neurol 2018;9:981.

11. Hicks D, Sarkozy A, Muelas N, et al. A founder mutation in anoctamin 5 is a major cause of limb-girdle muscular dystrophy. Brain 2011;134:171-182.

12. Piluso G, Politano L, Aurino S, et al. Extensive scanning of the calpain-3 gene broadens the spectrum of LGMD2A phenotypes. J Med Genet 2005;42:686-693.

13. Richard I, Broux O, Allamand V, et al. Mutations in the proteolytic enzyme calpain 3 cause limb-girdle muscular dystrophy type 2A. Cell 1995;81:27-40.

14. Kubisch C, Wicklein EM, Jentsch TJ. Molecular diagnosis of McArdle disease: revised genomic structure of the myophosphorylase gene and identification of a novel mutation. Hum Mutat 1998;12:27-32.

15. Piccolo F, Roberds SL, Jeanpierre M, et al. Primary adhalinopathy: a common cause of autosomal recessive muscular dystrophy of variable severity. Nat Genet 1995;10:243-245.

16. Duggan DJ, Gorospe JR, Fanin M, Hoffman EP, Angelini C. Mutations in the sarcoglycan genes in patients with myopathy. N Engl J Med 1997;336:618-624.

17. Savarese M, Di Fruscio G, Tasca G, et al. Next generation sequencing on patients with LGMD and nonspecific myopathies: findings associated with ANO5 mutations. Neuromuscul Disord 2015;25:533-541. 
18. Tsujino S, Shanske S, DiMauro S. Molecular genetic heterogeneity of myophosphorylase deficiency (McArdle's disease). N Engl J Med 1993;329:241-245.

19. Anichini A, Fanin M, Vianey-Saban C, et al. Genotype-phenotype correlations in a large series of patients with muscle type CPT II deficiency. Neurol Res 2011;33:24-32.

20. Vockley J, Rinaldo P, Bennett MJ, Matern D, Vladutiu GD. Synergistic heterozygosity: disease resulting from multiple partial defects in one or more metabolic pathways. Mol Genet Metab 2000;71:10-18.

21. Böhm J, Vasli N, Malfatti E, et al. An integrated diagnosis strategy for congenital myopathies. PLoS One 2013;8:e67527.

22. Manning BM, Quane KA, Lynch PJ, et al. Novel mutations at a CpG dinucleotide in the ryanodine receptor in malignant hyperthermia. Hum Mutat 1998;11:45-50.

23. Carss KJ, Stevens E, Foley AR, et al. Mutations in GDP-mannose pyrophosphorylase $\mathrm{B}$ cause congenital and limb-girdle muscular dystrophies associated with hypoglycosylation of $a$-dystroglycan. Am J Hum Genet 2013;93:29-41.

24. Sambuughin N, Sei Y, Gallagher KL, et al. North American malignant hyperthermia population: screening of the ryanodine receptor gene and identification of novel mutations. Anesthesiology 2001;95:594-599.

25. Vukcevic M, Broman M, Islander G, et al. Functional properties of RYR1 mutations identified in Swedish patients with malignant hyperthermia and central core disease. Anesth Analg 2010;111:185-190.

26. Sambuughin N, Nelson TE, Jankovic J, et al. Identification and functional characterization of a novel ryanodine receptor mutation causing malignant hyperthermia in North American and South American families. Neuromuscul Disord 2001;11:530-537.

27. Cagliani R, Fortunato F, Giorda R, et al. Molecular analysis of LGMD-2B and MM patients: identification of novel DYSF mutations and possible founder effect in the Italian population. Neuromuscul Disord 2003;13:788-795.

28. Sambuughin N, Holley H, Muldoon S, et al. Screening of the entire ryanodine receptor type 1 coding region for sequence variants associated with malignant hyperthermia susceptibility in the north american population. Anesthesiology 2005;102:515-521.

29. Taroni F, Verderio E, Dworzak F, Willems PJ, Cavadini P, DiDonato S. Identification of a common mutation in the carnitine palmitoyltransferase II gene in familial recurrent myoglobinuria patients. Nat Genet 1993;4:314-320.

30. Tammaro A, Di Martino A, Bracco A, et al. Novel missense mutations and unexpected multiple changes of RYR1 gene in 75 malignant hyperthermia families. Clin Genet 2011;79:438-447.

31. Duarte ST, Oliveira J, Santos R, et al. Dominant and recessive RYR1 mutations in adults with core lesions and mild muscle symptoms. Muscle Nerve 2011;44:102-108.
32. Gillies RL, Bjorksten AR, Davis M, Du Sart D. Identification of genetic mutations in Australian malignant hyperthermia families using sequencing of RYR1 hotspots. Anaesth Intensive Care 2008;36:391-403.

33. Fanin M, Fulizio L, Nascimbeni AC, et al. Molecular diagnosis in LGMD2A: mutation analysis or protein testing? Hum Mutat 2004;24:52-62.

34. Selcen D, Engel AG. Mutations in myotilin cause myofibrillar myopathy. Neurology 2004;62:1363-1371.

35. Venance SL. Approach to the patient with HyperCKemia. Continuum (Minneap MN) 2016;22:1803-1814.

36. Filosto $\mathrm{M}$, Tonin $\mathrm{P}$, Vattemi G, et al. The role of muscle biopsy in investigating isolated muscle pain. Neurology 2007;68:181-186.

37. Simmons Z, Peterlin BL, Boyer PJ, Towfighi J. Muscle biopsy in the evaluation of patients with modestly elevated creatine kinase levels. Muscle Nerve 2003;27: 242-244.

38. Prelle A, Tancredi L, Sciacco M, et al. Retrospective study of a large population of patients with asymptomatic or minimally symptomatic raised serum creatine kinase levels. J Neurol 2002;249:305-311.

39. Savarese M, Di Fruscio G, Torella A, et al. The genetic basis of undiagnosed muscular dystrophies and myopathies: results from 504 patients. Neurology 2016;87:71-76.

40. Wu L, Brady L, Shoffner J, Tarnopolsky MA. Next-generation sequencing to diagnose muscular dystrophy, rhabdomyolysis, and hyperCKemia. Can J Neurol Sci 2018;45: 262-268.

41. Papadopoulos C, LaforÊt P, Nectoux J, et al. Hyperckemia and myalgia are common presentations of anoctamin-5-related myopathy in French patients. Muscle Nerve 2017; 56:1096-1100.

42. Tarnopolsky M, Hoffman E, Giri M, Shoffner J, Brady L. Alpha-sarcoglycanopathy presenting as exercise intolerance and rhabdomyolysis in two adults. Neuromuscul Disord 2015;25:952-954.

43. Zatz M, Starling A. Calpains and disease. N Engl J Med 2005;352:2413-2423.

44. Vissing J, Barresi R, Witting N, et al. A heterozygous 21-bp deletion in CAPN3 causes dominantly inherited limb girdle muscular dystrophy. Brain 2016;139:2154-2163.

45. Fanin M, Anichini A, Cassandrini D, et al. Allelic and phenotypic heterogeneity in 49 Italian patients with the muscle form of CPT-II deficiency. Clin Genet 2012;82: 232-239.

46. Fontaine M, Kim I, Dessein AF, et al. Fluxomic assay-assisted diagnosis orientation in a cohort of 11 patients with myopathic form of CPT2 deficiency. Mol Genet Metab 2018;123:441-448. 


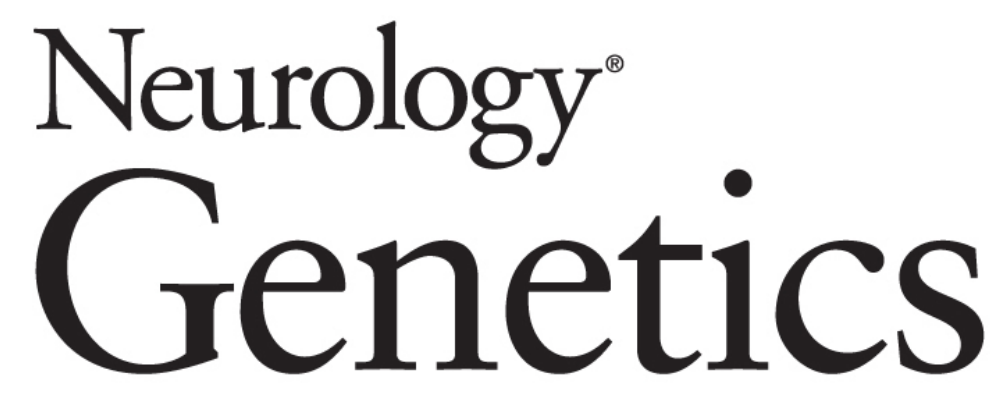

Next-generation sequencing approach to hyperCKemia: A 2-year cohort study Anna Rubegni, Alessandro Malandrini, Claudia Dosi, et al. Neurol Genet 2019;5;

DOI 10.1212/NXG.0000000000000352

This information is current as of August 16, 2019

\section{Updated Information \& Services}

References

Citations

Subspecialty Collections

Permissions \& Licensing

Reprints including high resolution figures, can be found at: http://ng.neurology.org/content/5/5/e352.full.html

This article cites 46 articles, 2 of which you can access for free at: http://ng.neurology.org/content/5/5/e352.full.html\#\#ref-list-1

This article has been cited by 3 HighWire-hosted articles: http://ng.neurology.org/content/5/5/e352.full.html\#\#otherarticles

This article, along with others on similar topics, appears in the following collection(s):

\section{All Genetics}

http://ng.neurology.org//cgi/collection/all_genetics

All Neuromuscular Disease

http://ng.neurology.org//cgi/collection/all_neuromuscular_disease Muscle disease

http://ng.neurology.org//cgi/collection/muscle_disease

Information about reproducing this article in parts (figures,tables) or in its entirety can be found online at:

http://ng.neurology.org/misc/about.xhtml\#permissions

Information about ordering reprints can be found online: http://ng.neurology.org/misc/addir.xhtml\#reprintsus

Neurol Genet is an official journal of the American Academy of Neurology. Published since April 2015, it is an open-access, online-only, continuous publication journal. Copyright Copyright (@) 2019 The Author(s). Published by Wolters Kluwer Health, Inc. on behalf of the American Academy of Neurology.. All rights reserved. Online ISSN: 2376-7839.

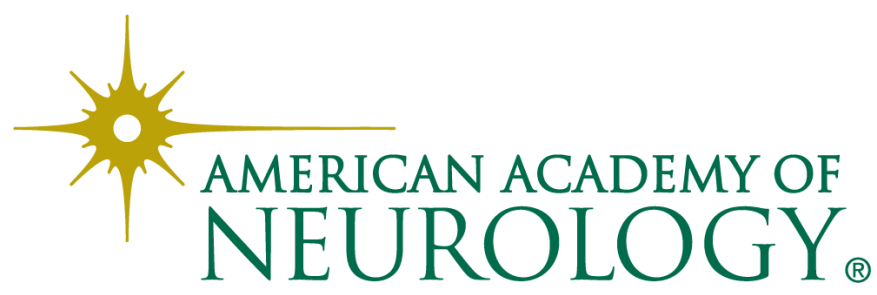

